\title{
A new species of the ginglymodian fish Isanichthys from the Late Jurassic Phu Kradung Formation, northeastern Thailand
}

Uthumporn Deesri, Komsorn Lauprasert, Varavudh Suteethorn, Kamonlak Wongko, and Lionel Cavin Acta Palaeontologica Polonica 59 (2), 2014: 313-331 doi: http://dx.doi.org/10.4202/app.2012.0013

A new ginglymodian fish, Isanichthys lertboosi, is described from the Phu Kradung Formation, north-eastern Thailand, a freshwater deposit of probable Late Jurassic age. The species is represented by four specimens, from the Phu Noi locality, associated with a rich fauna of sharks, turtles, crocodiles, and theropod and sauropod dinosaurs. One specimen is an isolated braincase, which provides characters rarely observed in extinct ginglymodians. The species is referred to the genus Isanichthys, a taxon originally described on the basis of a single specimen from the Phu Nam Jun locality, a slightly younger site approximately 75 $\mathrm{km}$ from Phu Noi. Isanichthys is mainly distinguished by frontals slightly narrower anteriorly than posteriorly, two anterior infraorbitals not in contact with the orbit, reduced preorbital region, and a small orbit and a cheek region completely covered by bones. The new species is characterized, among other characters, by a dermal component of the sphenotic visible on the cheek, one pair of extrascapulars plus a small median one, the presence of few suborbitals (ca. 4 or 6) arranged in one row, and a median dorsal row of scales with spine.

Comparisons with other ginglymodian taxa and a cladistic analysis indicates that Isanichthys (Lepidotes ) latifrons from the Late Jurassic of England, as well as probably Isanichthys (Lepidotes ) luchowensis from the Early or Middle Jurassic of Sichuan, China, form a clade with both Thai species of Isanichthys. The new species provides evidence of the high diversity of ginglymodian fishes in the Phu Kradung Formation and suggests a new hypothesis of phylogenetic relationships among extinct ginglymodians.

Key words: Actinopterygii, Holostei, osteology, braincase, phylogeny, Jurassic, south-east Asia.

Uthumporn Deesri [uthumporn_deesri@yahoo.com], Department of Biology, Faculty of Science, Mahasarakham University, Khamrieng, Kantharawichai District, Mahasarakham 44150, Thailand; Komsorn Laupresert [lauprasert@ gmail.com ], Department of Biology, Faculty of Science, Mahasarakham University, Khamrieng, Kantharawichai District, Mahasarakham 44150, Thailand and Palaeontological Research and Education Centre, Mahasarakham University, Khamrieng, Kantharawichai District, Mahasarakham 44150, Thailand; 
Varavudh Suteethorn [suteethorn@ hotmail.com], Palaeontological Research and Education Centre, Mahasarakham University, Khamrieng, Kantharawichai District, Mahasarakham 44150, Thailand; Kamonlak Wongko [uree40@ yahoo.com],

Department of Mineral Resources, Rama VI Road, Bangkok 10400, Thailand;

Lionel Cavin [lionel.cavin@ ville-ge.ch], Department of Geology and Palaeontology, Muséum d'Histoire naturelle, CP 6434, 1211 Genève 6, Switzerland.

This is an open-access article distributed under the terms of the Creative Commons Attribution License (for details please see creativecommons.org), which permits unrestricted use, distribution, and reproduction in any medium, provided the original author and source are credited.

For Full text $(1.732 .5 \mathrm{kB})$

$\mathrm{FOF}^{2}$ Supplementary file $(34.3 \mathrm{kB})$ 